\title{
Target Cell Count
}

National Cancer Institute

\section{Source}

National Cancer Institute. Target Cell Count. NCI Thesaurus. Code C96636.

The determination of the amount of target cells present in a sample. 